\title{
A report of three patients in whom the surgical closure of terminal branches of the external carotid arteries for treatment of migraine resulted in significantly reduced frequency of epileptic attacks
}

\author{
E Shevel, BDS, Dip MFOS, MB BCh \\ The Headache Clinic and Specialist Migraine Centre, Johannesburg, South Africa
}

Corresponding author: E Shevel (drshevel@headclin.com)

Three patients under treatment for grand mal epilepsy, and who were also suffering from chronic migraine, underwent vascular surgery for their migraine. A serendipitous benefit from the successful vascular surgery for migraine was a significant reduction in the frequency of their grand mal seizures.

S Afr Med J 2016;106(11):1084-1085. DOI:10.7196/SAMJ.2016.v106i1 1.10866

\begin{abstract}
This is the first report of a reduction in the frequency of grand mal attacks in patients who underwent surgical cauterisation of terminal branches of the external carotid artery to treat their migraine headaches. ${ }^{[1]}$ An unexpected consequence of the surgery was that all three patients experienced not only a dramatic reduction of their migraines, but also a significant reduction in the number of epileptic attacks. All three patients were under treatment for epilepsy at the time.

The treatment of choice at The Headache Clinic in Johannesburg for intractable migraine where the pain has been positively diagnosed to originate in the terminal branches of the external carotid artery is permanent surgical closure of the relevant vessels. ${ }^{[1]}$ Three patients diagnosed with migraine pain originating in the dilated terminal branches of the external carotid arteries, and who were also under treatment for grand mal epilepsy, underwent surgical cauterisation of the relevant vessels. All three reported that following the surgery, not only did they no longer suffer from previously refractory migraine, but that there had also been an immediate and dramatic reduction in the number of seizures that they experienced.
\end{abstract}

\section{Case reports and results}

Case 1

A 28-year-old woman presented in May 2011 with refractory chronic migraine that had started approximately 5 years earlier. She had also been under treatment for grand mal epilepsy for the previous 15 years. Her epilepsy was currently being treated at the neurology unit of a local hospital. Brain imaging showed no intracranial mass lesions. Her current medication was carbamazepine controlled release $400 \mathrm{mg}$ qid and lamotrigine $50 \mathrm{mg}$ bd, but she continued to experience an average of one grand mal seizure per day. The seizures were at times, but not always, triggered when she suffered a particularly severe migraine attack. The migraine pain was diagnosed as originating in the painfully dilated terminal branches of the external carotid artery. Consequently, the superficial temporal, frontal and occipital arteries bilaterally were surgically cauterised. The first surgical intervention was carried out on 9 May 2011, when the frontal arteries were cauterised. As the patient continued to experience headache pain, further surgery was carried out. On 31 May 2011, the superficial temporal and occipital arteries bilaterally were cauterised. She reported thereafter that she no longer suffered from headache pain. At follow-up on 9 February 2012, she reported that since the surgical intervention on 31 May 2011 she had not suffered a single seizure despite reducing her intake of carbamazepine to $200 \mathrm{mg}$ bd and lamotrigine to $50 \mathrm{mg}$ bd. As of 3 December 2015, 4 years later, she has remained free of seizures.

\section{Case 2}

A 32-year-old man presented in October 2012 with refractory chronic migraine that had started some time in his teens. He was also under treatment by a neurologist for grand mal epilepsy, which had started after surgery in 2000 for removal of an arachnoid cyst. On several occasions after the surgery he had had lumbar punctures and adjustments to his ventriculoperitoneal shunt, none of which had ever indicated increased intracranial pressure. He was on topiramate $75 \mathrm{mg}$ bd, clonazepam $1 \mathrm{mg}$ bd, sodium valproate $1200 \mathrm{mg}$ bd, carbamazepine $400 \mathrm{mg}$ bd and escitalopram $20 \mathrm{mg}$ daily. On this regimen he was experiencing an average of one seizure a week. Immediately following surgical cauterisation of the superficial temporal and occipital arteries bilaterally in October 2012, his seizures stopped. By the end of 2015, 3 years later, he had experienced no further seizures. His medication regimen remained the same.

\section{Case 3}

A 54-year-old woman presented in October 2014 with refractory chronic migraine, which had proved refractory to medication. The pain was bilateral, parietal and occipital, and also affected the vertex. When it was severe, it was accompanied by dizziness, nausea, photophobia, phonophobia and blurred vision. She was also under treatment by a neurologist for grand mal epilepsy, which had started 5 years previously. Her epilepsy medication consisted of sodium valproate controlled release, $500 \mathrm{mg}$ in the morning and $1000 \mathrm{mg}$ at night, and levetiracepam $500 \mathrm{mg}$ bd. Her epilepsy was poorly controlled on this regimen and she was experiencing an average of 10 seizures a day. Following surgical cauterisation of the superficial 
temporal and occipital arteries bilaterally, the frequency of her seizures dropped to three per week. At follow-up on 7 September 2015, she reported that she was still experiencing an average of three seizures per week. Her medication regimen remained unchanged.

\section{Discussion}

The internal carotid artery is unique in that a large outflow tract, the external carotid artery, is situated just at its origin. This disposition renders flow in the internal carotid dependent to a certain extent on flow characteristics of the external carotid arterial bed. ${ }^{[2]}$ It is possible that by reducing the blood flow to the extracranial tissues, there may be a resultant increase in blood flow to the intracranial tissues, due to shunting from the cauterised terminal branches of the external carotid to the internal carotid. This hypothesis is supported by the literature.

Bearing in mind that the results of animal studies cannot always be reliably extrapolated to humans, the reactivity of the neural vasculature in dogs is subordinate to that of the extraneural vasculature. ${ }^{[3]}$ In the
Asian monkey, there is a similar phenomenon, in that internal carotid blood flow is determined to a certain extent by the tonus in the external carotid tree. ${ }^{[4]}$ Of greater relevance though, is that bilateral external carotid ligations in both man and monkey have been shown to result in an increase in flow through the internal carotid arteries. ${ }^{[2]}$

The results presented in this article suggest that this surgery carried out in migraineurs with extracranial vascular pain may also be of benefit in some cases of epilepsy. 1. Shevel E. Vascular surgery for chronic migraine. Therapy 2007;4(4):451-456. DOI:10.2217/14750708.4.4.451
2. Abraham J, Shetty G, Chandy J. Preliminary observation on the hemodynamics of the internal carotid
artery following bilateral external carotid ligation in the monkey. J Neurosurg 1971;35(2):192-196.
DOI: 10.3171/jns.1971.35.2.0192
3. Abraham J, Margolis G, O'Loughlin JC, MacCarty WC, Jr. Differential reactivity of neural and
extra-neural vasculature. I. Role in the pathogenesis of spinal cord damage from contrast media in
experimental aortography. J Neurosurg 1966;25(3):257-269. DOI:10.3171/jns.1966.25.3.0257
4. Chandra R, Abraham J. Modification of neurotoxicity of methylglucamine diatrizoate (hypaque 75
per cent) by intra-arterial instillation of vasoactive drugs. Indian J Med Res 1969;57(7):1316-1324.

Accepted 19 July 2016. 